University of Wollongong

Research Online

Faculty of Engineering and Information

Faculty of Engineering and Information

Sciences - Papers: Part B

Sciences

2018

\title{
A digital image correlation study of a NiTi alloy subjected to monotonic uniaxial and cyclic loading-unloading in tension
}

Xiaohui Bian

University of Wollongong, xb124@uowmail.edu.au

Ahmed A. Saleh

University of Wollongong, asaleh@uow.edu.au

Elena V. Pereloma

University of Wollongong, elenap@uow.edu.au

Christopher H. J Davies

Monash University

Azdiar Adil Gazder

University of Wollongong, azdiar@uow.edu.au

Follow this and additional works at: https://ro.uow.edu.au/eispapers1

Part of the Engineering Commons, and the Science and Technology Studies Commons

Research Online is the open access institutional repository for the University of Wollongong. For further information contact the UOW Library: research-pubs@uow.edu.au 


\title{
A digital image correlation study of a NiTi alloy subjected to monotonic uniaxial and cyclic loading-unloading in tension
}

\author{
Abstract \\ This digital image correlation study details the mechanical behaviour and pattern evolution of the \\ transformation band of a 56Ni-44Ti wt\% shape memory alloy subjected to monotonic uniaxial and \\ loading-unloading cycles in tension. The broadened single inclined band front and multiple criss-crossing \\ patterns are found to relieve the in-plane moment caused by local shear strains and straighten the sample \\ edges during testing. The magnitude of the maximum local strain rate suggests its feasibility to \\ understand the direction and extent of the localised transformation. Specifically, the changes to the \\ maximum local strain rate during monotonic uniaxial tension are generally analogous to the stages in the \\ macroscopic stress-strain curve. The microstructure before and after mechanical testing was \\ characterised via electron back-scattering diffraction. Estimates of the kernel average misorientation \\ show that residual strains upon unloading are linked to high intragranular misorientation within the \\ original B2 grains and the remnant B19' variants.

\section{Disciplines} \\ Engineering | Science and Technology Studies

\section{Publication Details} \\ Bian, X., Saleh, A. A., Pereloma, E. V., Davies, C. J.H. \& Gazder, A. A. (2018). A digital image correlation \\ study of a NiTi alloy subjected to monotonic uniaxial and cyclic loading-unloading in tension. Materials \\ Science and Engineering A: Structural Materials: Properties, Microstructure and Processing, 726 102-112.
}




\title{
A digital image correlation study of a NiTi alloy subjected to monotonic uniaxial and cyclic loading-unloading in tension
}

Xiaohui Bian ${ }^{1,2}$, Ahmed A. Saleh¹, Elena V. Pereloma1,2, Christopher H.J. Davies ${ }^{3}$, Azdiar A. Gazder²

${ }^{1}$ School of Mechanical, Materials, Mechatronic and Biomedical Engineering, University of Wollongong, New South Wales 2500, Australia

2 Electron Microscopy Centre, University of Wollongong, New South Wales 2500, Australia

${ }^{3}$ Department of Mechanical and Aerospace Engineering, Monash University, Victoria 3800, Australia

\begin{abstract}
This digital image correlation study details the mechanical behaviour and pattern evolution of the transformation band of a $56 \mathrm{Ni}-44 \mathrm{Ti}$ wt.\% shape memory alloy subjected to monotonic uniaxial and loading-unloading cycles in tension. The broadened single inclined band front and multiple crisscrossing patterns are found to relieve the in-plane moment caused by local shear strains and straighten the sample edges during testing. The magnitude of the maximum local strain rate suggests its feasibility to understand the direction and extent of the localised transformation. Specifically, the changes to the maximum local strain rate during monotonic uniaxial tension are generally analogous to the stages in the macroscopic stress-strain curve. The microstructure before and after mechanical testing was characterised via electron back-scattering diffraction. Estimates of the kernel average misorientation show that residual strains upon unloading are linked to high intragranular misorientation within the original B2 grains and the remnant B19' variants.
\end{abstract}

Keywords: NiTi; shape memory alloys; digital image correlation; tension; martensite; electron backscattering diffraction 


\section{Introduction}

NiTi shape memory alloys subjected to monotonic uniaxial tension at low initial strain rates (between $\sim 10^{-3}$ and $\sim 10^{-4} \mathrm{~s}^{-1}$ ) typically present with macroscopic stress-strain curves comprising an initial elastic region, a stress plateau region (between $\sim 0.01-0.12$ engineering strains) followed by a rising stress region up to a maximum stress value [1-6]. Superelasticity is the ability of NiTi alloys to sustain repeated loading and unloading cycles within the stress plateau region; on account of the initial primitive cubic B2 (or austenite) phase undergoing a fully reversible, stress-induced transformation to monoclinic B19' (or martensite). Consequently, the study of martensitic transformation within the macroscopic stress plateau region necessitates employing in-situ techniques.

One of the effective techniques typically combined with mechanical testing is digital image correlation (DIC), which is an optical correlation technique that records and measures the displacement of random speckle patterns painted along the gauge length, in order to compute the evolution of surface strain during loading [7, 8]. Daly et al. [1] undertook the first in-situ DIC study of rolled, thin sheets of fully austenitic 52Ni-48Ti wt.\% alloy to quantify the evolution of localised strain fields up to the end of the macroscopic stress plateau region for one loading-unloading cycle in tension.

Since then, researchers have routinely applied DIC to characterise strain fields during the mechanical testing of thermo-mechanically processed NiTi alloys [3, 9-14]. For example, DIC investigations revealed the development of a region of strain concentration upon deviation from linearity towards the end of the elastic region; suggesting the initiation of phase transformation just prior to the onset of the macroscopic stress plateau [1]. Thereafter, further localisation in the strain fields results in Lüders/transformation band(s) that propagate along the gauge length with higher macroscopic tensile strains $[1,3,11,12,14-16]$. The bands are typically inclined at $\sim 60^{\circ}$ with respect to the loading direction $[12,17-21]$ and divide the gauge length into domains with varying local axial strain extrema comprising fully martensitic and as-yet untransformed B2 regions $[11,12,14,15]$. The axial strain values within the mobile band fronts do not saturate but record a continuous increase with higher macroscopic tensile strains [1, 14].

While a single band propagates along the gauge length during monotonic uniaxial tension, multiple criss-crossing bands develop during both cyclic loading-unloading in tension and fatigue tests $[1,9,11,13,15,19]$. However, the mechanism behind the forward propagation of the mobile front of single bands along the gauge length and the switching from single to multiple criss-crossing bands is unclear. To-date, a couple of studies have suggested that shear strains lead to an in-plane moment, which in turn may influence the properties of the mobile band front $[16,19]$.

Fatigue testing of NiTi alloys typically involves repeated cycles of loading up to the end of the macroscopic stress plateau region and then unloading back to zero load. Such investigations reported increases in the irreversible strain component (through highly accumulated dislocation densities and the formation of stable martensite variants with successive cycles) contributing to an increase in the 
residual strain upon unloading and a progressive degradation in superelasticity involving the retention of an increasing phase fraction of B19' [14, 15, 22-29].

However, the typical fatigue test as described above is markedly different from loadingunloading cycles, involving small increments to the macroscopic strain per cycle, within and up to the end of the macroscopic stress plateau region. Although the latter type of mechanical tests are less frequently reported, it is necessary to enable: (i) an understanding of localised deformation accumulation through axial/transverse/shear strain pattern evolution via incremental increases to the macroscopic strain and, (ii) studying the degradation in superelasticity as a function of the overall stress versus strain response with successive cycles.

While the changes in the DIC-based axial strains during mechanical testing have been catalogued, the local strain rate, which affects the direction and extent of phase transformation within the macroscopic stress plateau region, has only been reported for a select macroscopic tensile strain [3]. It was found that the local strain rate is positive only within the narrow propagating band front whereas it is close to zero elsewhere along the gauge length [3]; indicating that phase transformation is confined within the band front area. In addition, the maximum local strain rate for the select time step is approximately an order of magnitude higher than the macroscopic strain rate, which suggests its feasibility as a descriptive indicator of the rate of localised transformation occurring at each time step [3].

With the above outlook in mind, the present DIC study aims to detail the mechanical behaviour of a NiTi shape memory alloy subjected to monotonic uniaxial and loading-unloading cycles in tension. For the latter test type, loading-unloading cycles are undertaken with small increments to the macroscopic tensile strain per cycle, within and up to the end of the macroscopic stress plateau region. In particular, a systematic description of the mechanisms of single transformation band front broadening and criss-crossing patterns is provided. Furthermore, the evolution of maximum local strain rate with macroscopic strain is explored. Microstructure comparisons before and after mechanical testing were undertaken via electron back-scattering diffraction (EBSD).

\section{Experimental procedure}

An as-received 56Ni-44Ti wt.\% rod produced by Nitinol Devices and Components Inc. was cut into smaller $\varnothing 10 \times 70 \mathrm{~mm}^{2}$ rods by electro-discharge machining. These small rods were encapsulated in a quartz tube under an Ar atmosphere, annealed at $700{ }^{\circ} \mathrm{C}$ for $120 \mathrm{~s}$ in a muffle furnace and immediately water quenched. The resultant microstructure comprised a single B2 phase with an average grain size of $\sim 10 \pm 5.9 \mu \mathrm{m}$.

The annealed rods were electro-discharge machined into 18 (gauge length) $\times 5$ (width) $\times 1$ (thickness) $\mathrm{mm}^{3}$ flat dog-bone samples with their gauge lengths parallel to the rod axial direction. For DIC measurements, a random speckle pattern was created by spraying a black background and white spots along the entire gauge length prior to tension. 
Two types of mechanical tests involving monotonic uniaxial tension and cyclic loadingunloading were performed at room temperature using a computer-controlled servo-hydraulic $100 \mathrm{kN}$ Instron 1341 universal testing machine operating at a crosshead speed of $0.48 \mathrm{~mm} \cdot \mathrm{min}^{-1}$; which corresponds to an initial strain rate of $1 \times 10^{-4} \mathrm{~s}^{-1}$.

During uniaxial tension, the sample was monotonically loaded beyond the macroscopic stress plateau region, well into the slowly rising macroscopic stress region and then unloaded; in order to cover all characteristic deformation stages. Cyclic loading-unloading in tension was undertaken in 0.005 engineering strain increments per cycle, for a total of 20 cycles. The loading-unloading speeds were the same.

The force data from the load cell was transmitted as an analogue signal to the Instron and DIC computers; both of which were synchronised to record concurrently time-stamped stress readings and image frames. DIC data contained recording images of the random speckles painted along the gauge length on a Dantec Dynamics Q-400 system comprising two close circuit digital cameras operating at 5 frames per second for the duration of the mechanical tests.

Thereafter, an in-house developed Matlab script was used to compute the macroscopic engineering strain. The script identifies the grid locations of all speckles centroids along the gauge length span for the first image frame (used as the reference image) and then calculates their displacements in successive frames. For each successive frame, the average axial displacement was used to compute the macroscopic average engineering strain $(\bar{\varepsilon})$. The evolution in axial and shear strains along the entire gauge length is shown using contour maps computed using the Istra-4D software suite.

Microstructure characterisation was undertaken by polishing the flat dog-bone sample face up to the $1 \mu \mathrm{m}$ diamond stage and then electro-polishing at room temperature using a Struers Lectropol-5 operating at $20 \mathrm{~V}(\sim 1.8 \mathrm{~A})$ for $180 \mathrm{sec}$ with an electrolyte mixture comprising $73 \%$ ethanol $+10 \%$ butoxyethanol $+9 \%$ water $+8 \%$ perchloric acid by volume.

EBSD was performed on a JEOL JSM-7001F field emission gun-scanning electron microscope operating at $15 \mathrm{kV}, \sim 6.5 \mathrm{nA}$ probe current $12 \mathrm{~mm}$ working distance and fitted with an Oxford Instruments Nordlys-II(S) camera interfacing with the AZtec acquisition software suite. A magnification of $150 \times$ and a step size of $0.5 \mu \mathrm{m}$ was employed for the annealed sample whereas a magnification of $2000 \times$ and a step size of $0.03 \mu \mathrm{m}$ was used for the two samples after mechanical testing.

The EBSD maps were post-processed using the Oxford Instruments Channel-5 software suite. The maps were cleaned by removing the wild spikes, cyclic extrapolation of zero solutions to five neighbours and thresholding the band contrast to delineate unindexed regions. In this study, phases are superimposed on the band contrast map such that the red, teal and white denote the B2 and B19' phases and unindexed regions, respectively. For all calculations, misorientations between $2^{\circ}-15^{\circ}$ and greater than $15^{\circ}$ are classified as low (LAGBs, in silver) and high (HAGBs, in black) -angle boundaries, 
respectively. The kernel average misorientation (KAM) maps were computed using a $3 \times 3$ square filter and a critical subgrain angle of $2^{\circ}$.

\section{Results}

In this study, the macroscopic engineering stress versus strain responses are plotted and analysed, as they are commonly reported in the literature $[1,3,13,30]$. In the following paragraphs, all strains are axial unless explicitly stated otherwise. The macroscopic average axial engineering strain $(\bar{\varepsilon})$ is referred to as the macroscopic strain whereas the local axial engineering strains and strain rates along the gauge length are referred to as local strains and strain rates, respectively.

\section{$\underline{3.1 \text { Monotonic uniaxial tension }}$}

Fig. 1a depicts the macroscopic engineering stress $(\sigma)$ versus average strain $(\bar{\varepsilon})$ curve during monotonic uniaxial tension. For the points highlighted by red circles, the corresponding sequence of full-field DIC images with varying scale bars are given in Fig. 2. The numbers indicate characteristic stages at various macroscopic strain values whereas letters denote intermediate points between stages.

The present 56Ni-44Ti wt.\% alloy returned an apparent elastic modulus of $72.5 \mathrm{GPa}$ (calculated between 0-270 MPa and 0-0.0035 macroscopic strain) for the B2 phase, which is within the 40-90 GPa range reported in the literature [5]. Fig. 1a(inset) shows that the macroscopic stress: (i) increases approximately linearly up to $295 \mathrm{MPa}$ (points (1) to (a)), (ii) then deviates from linearity causing an $\sim 20$ GPa drop in elastic modulus (points (b) to (2)), (iii) reaches a maximum value of $\sim 325 \mathrm{MPa}$ (point (2)), and (iv) thereafter reduces with the onset of the macroscopic stress plateau region at $\sim 295 \mathrm{MPa}$ and 0.0058 macroscopic strain (points (2), (c), (d) and (3)). The macroscopic stress plateau region extends up to $\sim 320 \mathrm{MPa}$ and 0.0944 macroscopic strain (point (4)) followed by a rising macroscopic stress region (points (4) to (5)). 


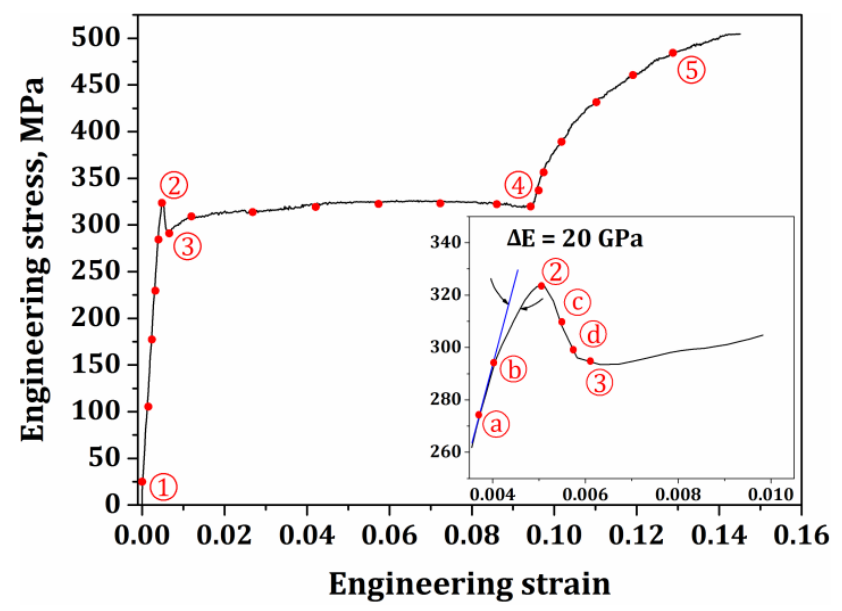

(a)

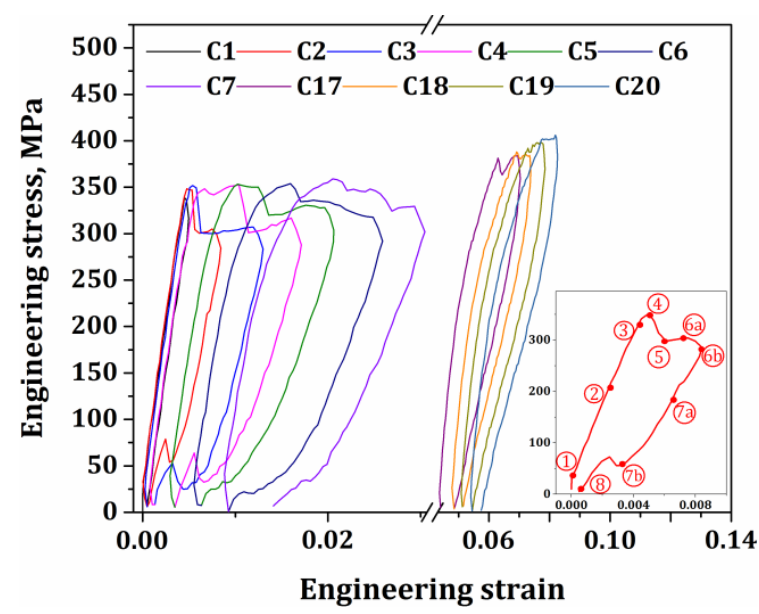

(b)

Figure. 1. The macroscopic stress versus strain curve during (a) monotonic uniaxial tension and (b) cyclic loading-unloading in tension. C1-C20 designate the cycle number. The red points indicate strain values at which DIC strain contours are provided; the numbers and letters indicate characteristic stages of deformation.

Comparing the above deformation stages with the DIC images in Fig. 2, it is seen that within the elastic region, the macroscopic strain fluctuates while increasing gradually (points (1) to (a)). The deviation from linearity (points (b) to (2)) is accompanied by local strain accumulation; suggesting the nucleation of a B19' domain at the lower end of the gauge length near the end of the elastic region. Upon reaching the maximum stress, the subsequent reduction in stress corresponds to the propagation of the local strain accumulation across the sample width, resulting in a transformation band and stress relaxation in the sample bulk (points (2), (c), (d) and (3)). Point (3) corresponds to the onset of the macroscopic stress plateau region and marks the full development of the transformation band across the sample width (the local strain within the band is 0.05 whereas the macroscopic strain is only 0.0058 ) and the beginning of its forward propagation along the gauge length. It follows that the transformation band divides the gauge length between transformed and untransformed regions.

Within the macroscopic stress plateau region (points (3) to (4)), the mobile upper and lower band fronts propagate upwards and downwards along the gauge length, respectively, suggesting the progressive stress-induced phase transformation of B2 to B19'. Following the end of the macroscopic stress plateau region (points (4) to (5)), the macroscopic stress begins to rise suggesting that deformation accommodation by the B19' phase proceeds uniformly.

However, it should be pointed out that the end of the macroscopic stress plateau region (point (4)) is not accompanied by the completion of the strain localisation process; such that the small remnant pockets of the B2 phase finally transform at 0.0979 macroscopic strain. Once transformation saturates, deformation accommodation proceeds uniformly along the gauge length. 

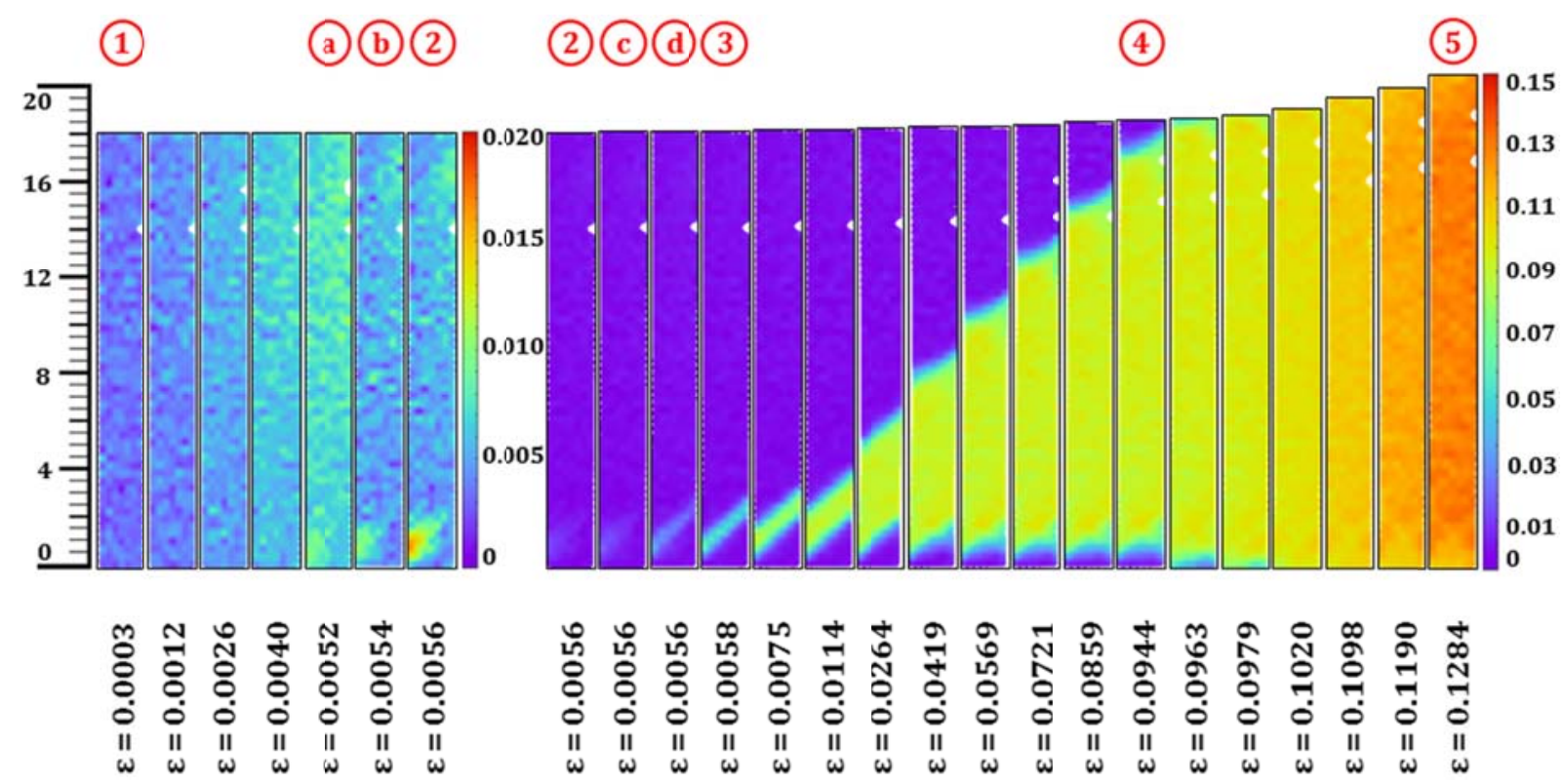

Figure. 2. Selected DIC strain contours corresponding to the red points in Fig. 1a. 1-2: deformation within the elastic region, 2-3: the initiation and development of the localised transformation band, 3-4: propagation of the transformation band within the macroscopic stress plateau region, 4-5: deformation within the slowly rising macroscopic stress region.

\section{1(a) Local strain fields and strain rate distributions during forward propagation of the transformation} band

To highlight the effect of strain localisation, Fig. 3a illustrates the variation in local strain along the centre line of the gauge length for select macroscopic strains.

Within the elastic region (0.0051 macroscopic strain), the local strain imposed on the B2 phase is approximately uniform throughout the gauge length. Between 0.0056 and 0.0114 macroscopic strains, an area of higher strain nucleates and expands; indicating phase transformation within the transformation band. As the band propagates along the gauge length between 0.0114 and 0.0944 macroscopic strains, the local strain values within the untransformed region correspond to the onset value of the macroscopic stress plateau region whereas, the values within the band correspond to the end of macroscopic stress plateau region. Once the forward propagation of the transformation band along the gauge length is complete (0.0979 macroscopic strain), the local strain along the gauge length is approximately uniform and thereafter, increases uniformly (0.1284 macroscopic strain).

In Fig. 3b, an analysis of local strains (solid lines) and strain rates ( $\dot{\varepsilon}$, dashed lines) along the centre line of the gauge length is presented for macroscopic strains 0.0085 (blue lines) and 0.0608 (black lines). These macroscopic strains are between points 3 and 4 in Figs. 1a and 2, such that 0.0085 is a macroscopic strain just after the onset of the macroscopic stress plateau region whereas 0.0608 is a macroscopic strain towards the end of the macroscopic stress plateau region when the forward propagation of the transformation band is well underway. 
In both cases, and comparing with the local strain values, the local strain rates are: (i) close to zero within the transformed and untransformed regions and, (ii) non-zero in the local strain transition zone between the transformed and untransformed regions.

The range of non-zero strain rates span that region of the gauge length within which transformation is current for any macroscopic strain within the macroscopic stress plateau region. The maximum local strain rate corresponds to the mobile front of the transformation band and is located in the middle of the local strain transition zone. It follows that changes in the position of the maximum local strain rate can be used to track the overall direction of forward propagation of the transformation band along the gauge length for the macroscopic strains within the macroscopic stress plateau region.

As per Ref. [3], Fig. 3c is a plot of the maximum local strain rates $\left(\dot{\varepsilon}_{M}\right)$ with respect to the macroscopic strain. The changes to the maximum strain rates are generally analogous to the stages seen in the macroscopic stress-strain curve (Fig. 1a). After strain localisation but prior to transformation band formation (between 0.0054 and 0.0056 macroscopic strains or Region 1 in Fig. $3 c)$, the maximum local strain rate $\dot{\varepsilon}_{\mathrm{M}}$ is relatively low $\left(\sim 1.2 \times 10^{-4} \mathrm{~s}^{-1}\right)$ and is of the same order as the macroscopic strain rate $\left(1 \times 10^{-4} \mathrm{~s}^{-1}\right)$. Transformation band formation is reflected as a sharp increase of the maximum local strain rate; with the latter 40 times higher than the macroscopic strain rate (point 2). During the forward propagation of the transformation band within the macroscopic stress plateau region (between points 3 and 4), $\dot{\varepsilon}_{\mathrm{M}}$ fluctuates between $1.4-2.3 \times 10^{-3} \mathrm{~s}^{-1}$ at an average of $\sim 1.7 \times 10^{-3} \mathrm{~s}^{-1}$, and is 15 times higher than the macroscopic strain rate or one time lower than the local strain rate recorded at band formation. In the rising macroscopic stress region (between points 4 and 5), the magnitude of the local strain rate is once again similar to the macroscopic strain rate at point 1 . 


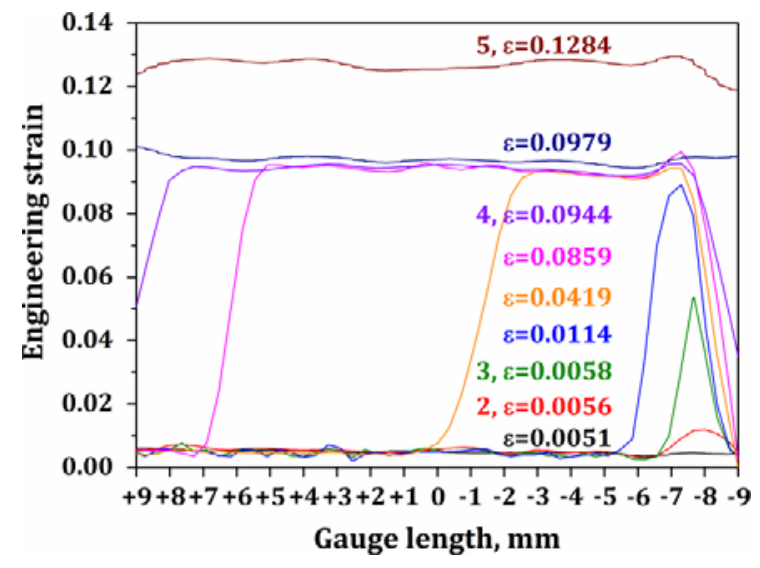

(a)

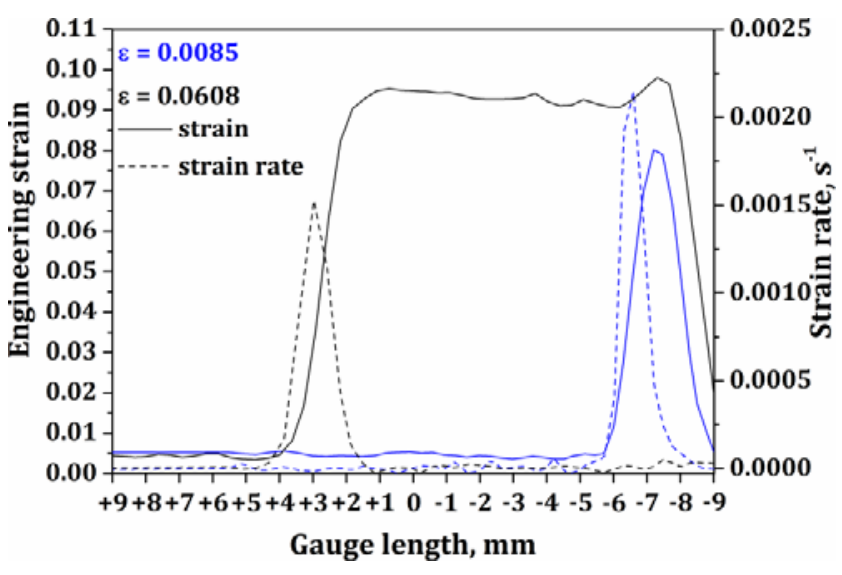

(b)

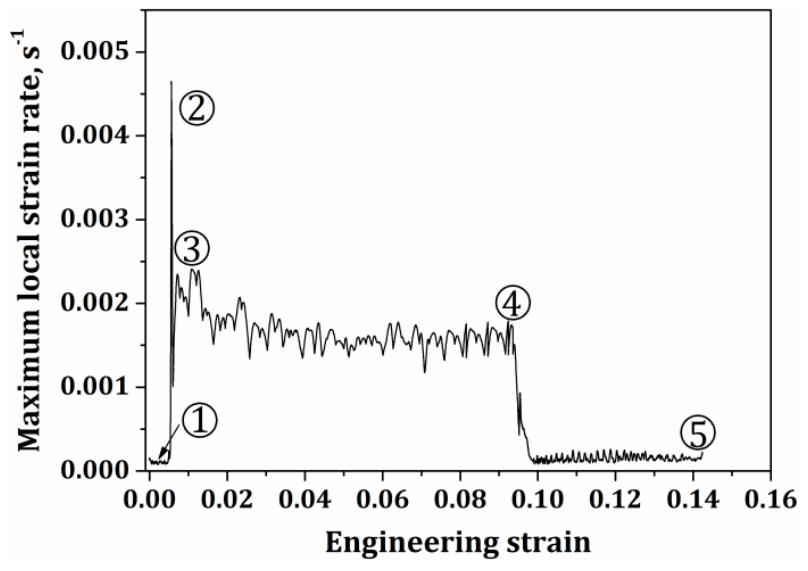

(c)

Figure. 3. (a) Local strain fields along the centre line of the gauge length for selected macroscopic strains (according to Fig. 2). (b) Local strain and strain rate distributions at macroscopic strains of 0.0085 (blue) and 0.0608 (black). In the figure transformation is progressing from right to left (following the upper band front in Fig. 2). (c) Evolution of the maximum local strain rates extracted from the middle of the moving band front. The numbers signify characteristic stages of deformation (according to Figs. 1a and 2).

\section{1(b) Characteristics of the mobile front of the transformation band}

As calculated in the literature [12, 17-21] and shown in Fig. 4a, the mobile, upper front of the transformation band is inclined at angle $(\eta)$ of $\sim 60^{\circ}$ to the sample axial length. Within the macroscopic stress plateau region, the sample does not undergo uniform elongation or narrowing along its axial and transverse directions, respectively. For example, at 0.0264 macroscopic strain, a local $\sim 2^{\circ}$ kink is observed at the intersection of the mobile, upper front of the transformation band and the sample edge. The kink disappears by 0.0721 macroscopic strain.

In Figs. 4b and 4c, the local shear strains along the gauge length at the left (L, green lines), centre (C, black lines) and right ( $R$, blue lines) of the sample width are presented for 0.0264 and 0.0721 macroscopic strains, respectively. While the centre line is representative of the middle of the sample width, the exact positions of the left and right lines were selected to cover distinct shear variations 
across the mobile band fronts. Analogous to the maxima in local strain rates, the maxima in local shear strains along the left, centre and right lines correspond to: (i) the position of forward propagation of the mobile fronts of transformation band and, (ii) the regions of the gauge length within which transformation is current for any macroscopic strain within the macroscopic stress plateau region. Furthermore, the distribution of local shear strains also serves to highlight the geometry of the transformation band. The left and right lines show maxima and minima in shear strain values for approximately the same position throughout the gauge length.

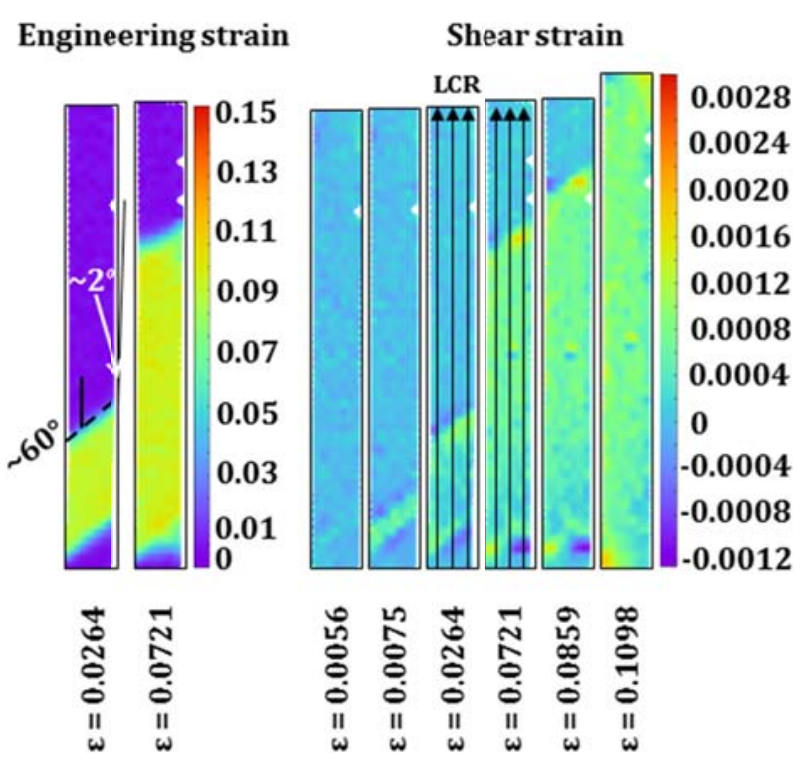

(a)

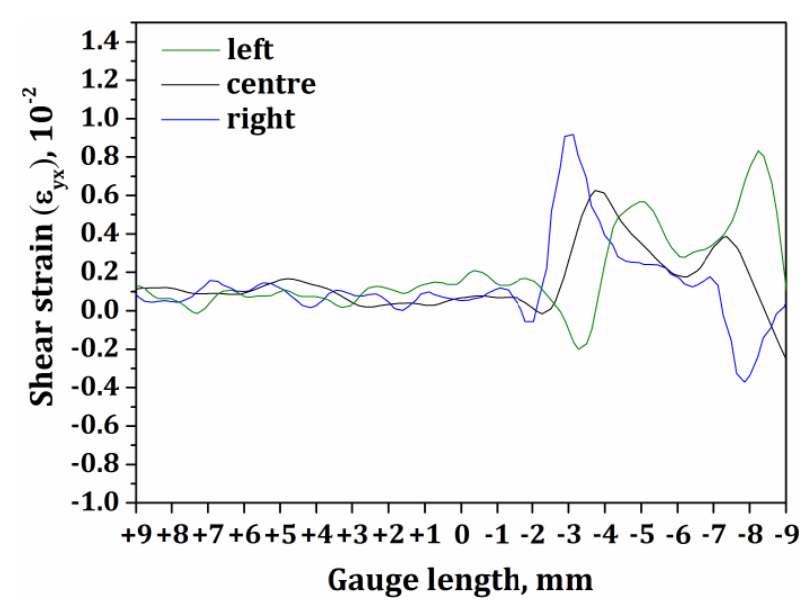

(b)

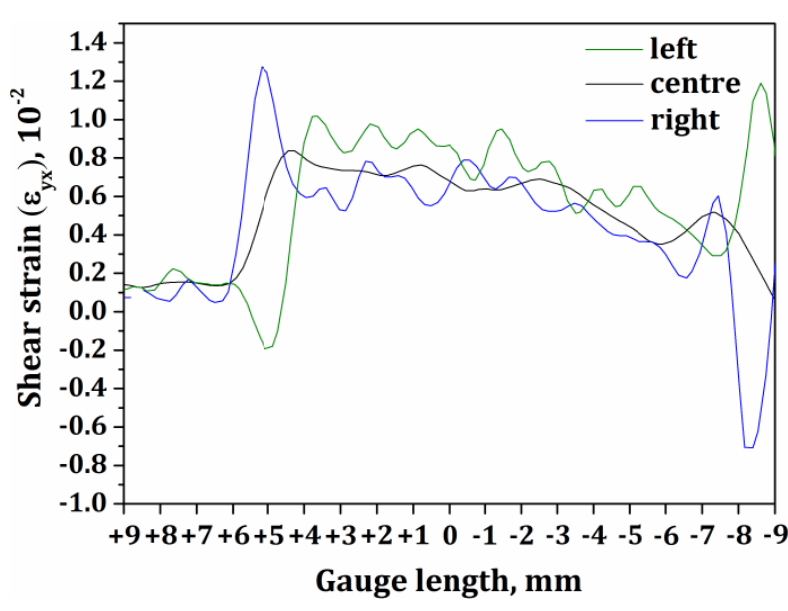

(c)

Figure. 4. (a) The inclined band front, local kink (0.0264 macroscopic strain) and broadened band front (0.0721 macroscopic strain) reflected on axial strain contours (left); representative shear strain contours are shown (right). Shear strain distribution along left (L), centre (C) and right (R) lines in (a) is shown for macroscopic strains of 0.0264 (b) and 0.0721 (c). 


\section{$\underline{3.2 \text { Cyclic loading-unloading in tension }}$}

Fig. 1b depicts the macroscopic engineering stress $(\sigma)$ versus average strain $(\bar{\varepsilon})$ curve during cyclic loading-unloading in tension. Although the loading-unloading tests were undertaken in 0.005 macroscopic strain increments per cycle (C) for a total 20 cycles, Fig. 1b shows cycles C1 to C7 followed by cycles $\mathrm{C} 17$ to $\mathrm{C} 20$, to avoid cluttering the plot.

The corresponding sequence of full-field DIC images with varying scale bars are given in Fig. 5 for cycles C2 to C5, C7 and C20. The numbers and letters indicate characteristic stages of deformation at various macroscopic strain values. These include: (1) points at the start and (2) middle of the elastic region, (3) the mid-point of deviation from elastic linearity, (4) the maximum stress point, (5) the point of onset of the macroscopic stress plateau region after stress reduction is complete, (6a) is the mid-point of a single macroscopic stress plateau region or just after stress relaxation and the onset of a second macroscopic stress plateau region, $(6 \mathrm{~b})$ is the end point of the macroscopic stress plateau region, $(7 \mathrm{a}, 7 \mathrm{~b})$ points that are one and two -thirds through the unloading region and, (8) the point at zero load after unloading is complete. An example of selected points is shown in Fig. 1b(inset), using the macroscopic stress versus strain curve of cycle C2. As the macroscopic stress versus strain response has changed from plateau type to curvilinear elastic with cycles, points (5) and (6a, 6b) for cycle C20 were selected at strain values where stress fluctuations are concurrent due to phase transformation occurring between the existing transformation bands.

During cycle C1, the sample was unloaded within the elastic region just before reaching the maximum stress point (compare Figs. 1a and 1b). Thus, the sample was cyclically loaded-unloaded in tension within the macroscopic stress plateau region from cycle C2 onwards. Fig. 5a clearly shows the development of local strain accumulations at points (3) and (4); suggesting the nucleation of B19' at the top and bottom ends of the gauge length towards the end of the elastic region.

Following the maximum stress and its subsequent decrease, the onset of the macroscopic stress plateau region corresponds to the propagation of the local strain accumulations across the sample width at the top and bottom ends of the gauge length as seen in point (5). Points (6a) and (6b) indicate the forward propagation of the top transformation band upwards and downwards along the gauge length; with the transformation band dividing the gauge length between transformed and untransformed regions.

During unloading, the macroscopic stress versus strain curve records a deviation from linearity, a stress increment followed by a linear reduction in stress to zero load (Fig. 1b). The stress increment is indicative of two mechanisms, i.e. - the elastic unloading of the B2 and B19' phases and reverse transformation of B19' back to B2. In Fig. 5a, this is reflected in points (7a) and (7b) by the reverse propagation of the top transformation band. Upon unloading to zero load, approximately zero residual strain is observed (point (8)).

During cycle C3 (Fig. 5b), points (6a) and (6b) indicate the forward propagation of the bottom transformation band upwards and downwards along the gauge length. Similar to cycle C2, during 
unloading, the reverse propagation of the bottom transformation band occurs (points (7a) and (7b)). Upon unloading to zero load, a residual strain of 0.01 is retained within the top and bottom transformation bands (point (8)).

In cycle C4 (Fig. 1b), the elastic region and the maximum stress are immediately followed by the macroscopic stress plateau region. No reduction in stress before the onset of the macroscopic stress plateau region occurred. With the onset of the macroscopic stress plateau region, Fig. 5c, points (4) and (5) shows that the majority of strain accommodation occurred within the pre-existing bottom transformation band.

Following this, point (6a) evidences the forward propagation of the bottom transformation band while the top transformation band propagates across the sample width and records higher strain values. The latter results in stress relaxation in the sample bulk, which is recorded as a reduction in the macroscopic stress prior to the onset of a new macroscopic stress plateau region (Fig. 1b). By the end of this new stress plateau region (point (6b)), forward propagation of the top and bottom transformation bands occurs. During unloading, the reverse propagation of the top and bottom transformation band takes place (points (7a) and (7b)) such that at zero load a residual strain of 0.025 is retained within the bottom transformation band (point (8)).

In cycle C5 (Fig. 1b), the non-linearity in the elastic region is ascribed to the residual strain carried over from the previous four cycles. Similar to cycle C4, the maximum stress is immediately followed by a macroscopic stress plateau region; which corresponds to points (3) and (4) with strain accommodation occurring within the pre-existing top and bottom transformation bands. Points (5) and (6a) show the development and propagation of a new transformation band inclined at $\sim 60^{\circ}$ crisscrossing the pre-existing bottom transformation band inclined at $\sim 120^{\circ}$. Once again, this results in stress relaxation in the sample bulk, which is recorded as a reduction in the macroscopic stress prior to the onset of a new macroscopic stress plateau (Fig. 1b). Up to the end of this new stress plateau region (point (6b)), forward propagation of all three transformation bands located at the top and bottom of the gauge length occurs. This is followed by reverse propagation on unloading (points (7a) and (7b)). At zero load, a residual strain of 0.04 is retained within the top and bottom transformation bands (point (8)).

During cycle C7 (Figs. 1b and 5e), greater non-linearity in the elastic region is ascribed to the higher residual strains carried over from previous cycles. This results in greater strain accommodation within pre-existing transformation bands and the further development and forward propagation of new, criss-crossing bands from points (3) to (6b). Unloading results in the reverse propagation of all bands (points (7a) and (7b)) and the retention of higher residual strains at zero load (point (8)).

Between cycles C1 and C7, the macroscopic stress versus strain curve shows a distinct change from manifesting one or two stress plateau(s). Alternatively, between cycles C17 and C20, the macroscopic stress versus strain curve shows a curvilinear elastic region followed by stress fluctuations (Fig. 1b). In cycle C20 (Fig. 5f), the curvilinearity upon loading is ascribed to strain 
accommodation mainly occurring within the criss-crossing transformation bands remained throughout the gauge length (points (1) to (4)), which is a result of increasingly accumulated residual strains from previous cycles. Additional phase transformation occurs in regions between the crisscrossing transformation bands (points (5) to (6b)), which leads to stress fluctuations following the elastic region. During unloading, local strain is decreased within the transformation bands but no reverse propagation of the bands occurs. This is due to the increased residual strains distributed uniformly along the gauge length.

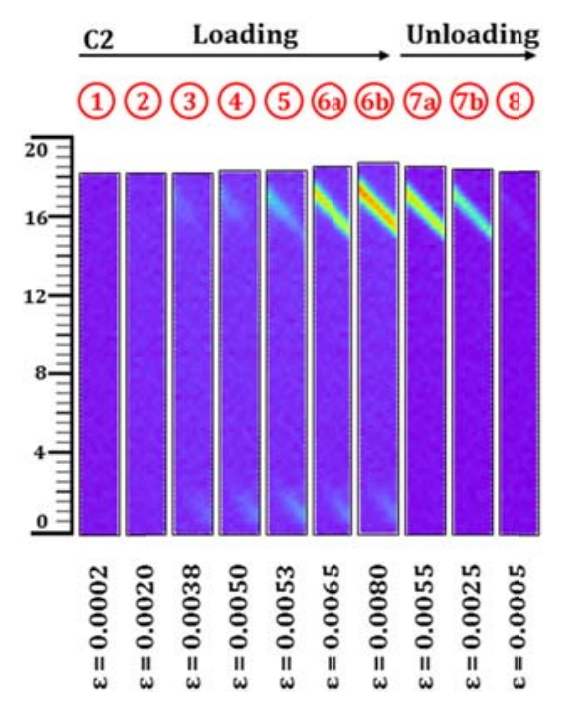

(a)

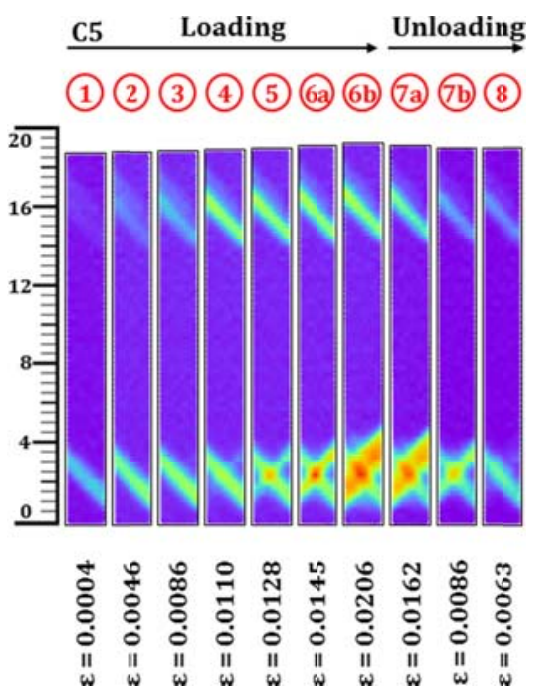

(d)

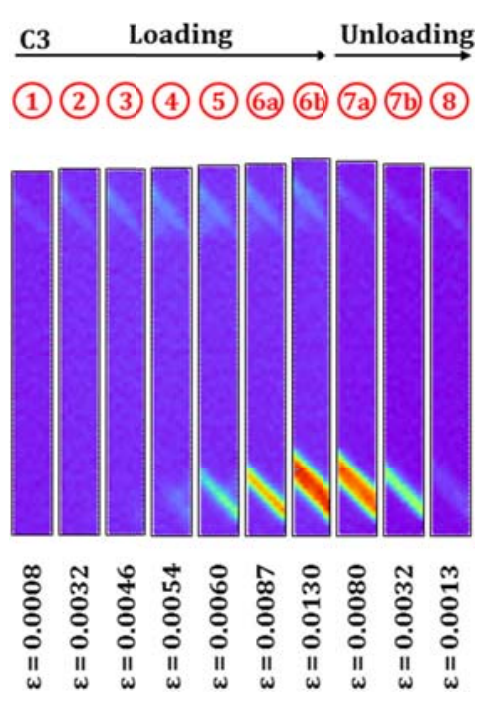

(b)
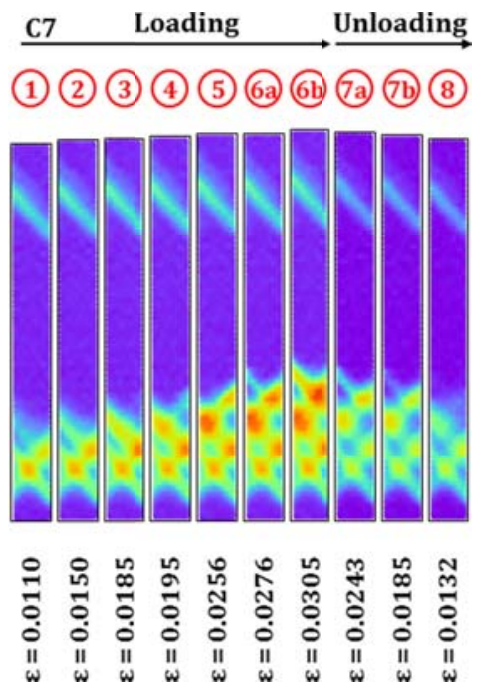

(e)

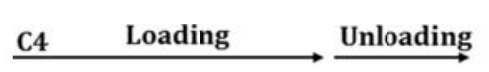

(1) (2) (3) (4) (5) (62) (6) (27) (76) (8)
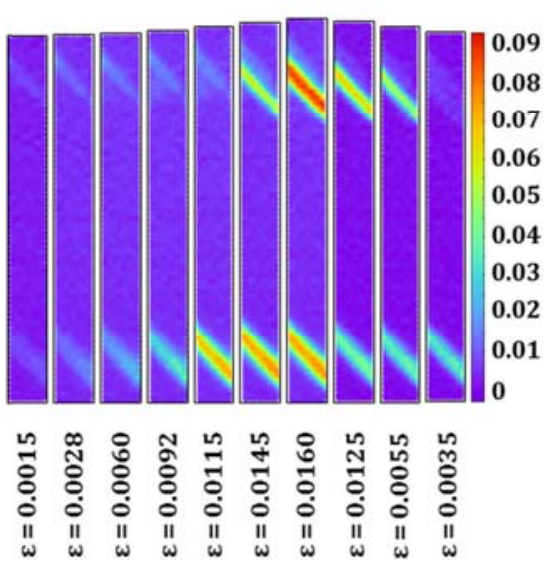

(c)
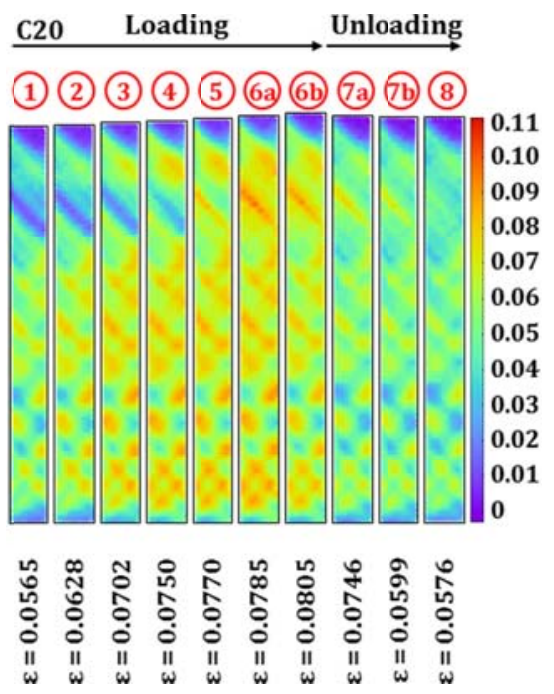

(f)

Figure. 5. DIC strain contours for selected loading-unloading cycles (with macroscopic strains shown): cycles C2 to C5 (a-d), cycle C7 (e) and cycle C20 (f). The numbers and letters indicate corresponding loading stages described in the text. 
3.2(a) Local strain fields and strain rate distributions during forward and reverse propagation of transformation bands

Figs. 6 and 7 illustrate the variation in local strain and strain rate along the left, centre and right of the gauge length for select macroscopic strains (corresponding to points (1) to (8) in Fig. 5) for cycles $\mathrm{C} 4$ and $\mathrm{C7}$. While the centre line is representative of the middle of the sample width, the exact positions of the left and right lines were selected to cover distinct local strain and strain rate variations across the mobile band fronts. Cycle $\mathrm{C} 4$ is the last cycle comprising single transformation bands located at the top and bottom of the gauge length whereas cycle C7 is representative of the ongoing development and propagation of criss-crossing transformation bands.

Analogous to Figs. 3a and 3b, during loading (points (1) to (6b)), an increase in local strain values occurs. The transition zones of local strains correspond to the maxima in local strain rates along the left, centre and right lines. In turn, they are correlated to: (i) the position of forward propagation of the mobile fronts of transformation bands and, (ii) the regions of the gauge length within which transformation is current for any macroscopic strain. During unloading (points (7a) to (8)), a decrease in value of the local strain maxima (i.e. - within a transformation band) is accompanied by a change in sign of the strain rate from positive to negative. They correspond to the reverse propagation of the mobile fronts of transformation bands.
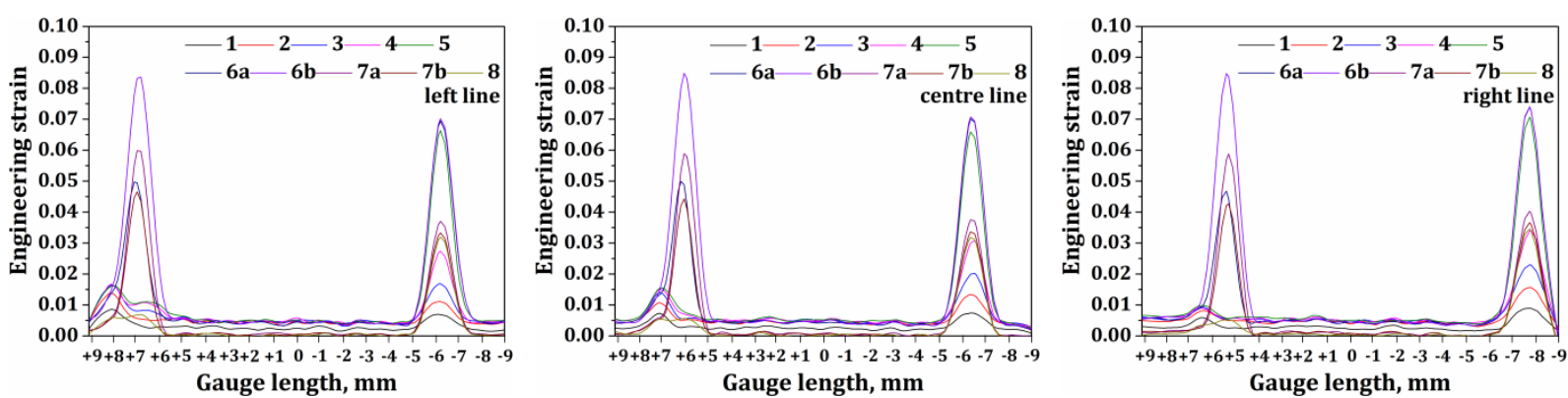

Gauge length, $\mathrm{mm}$
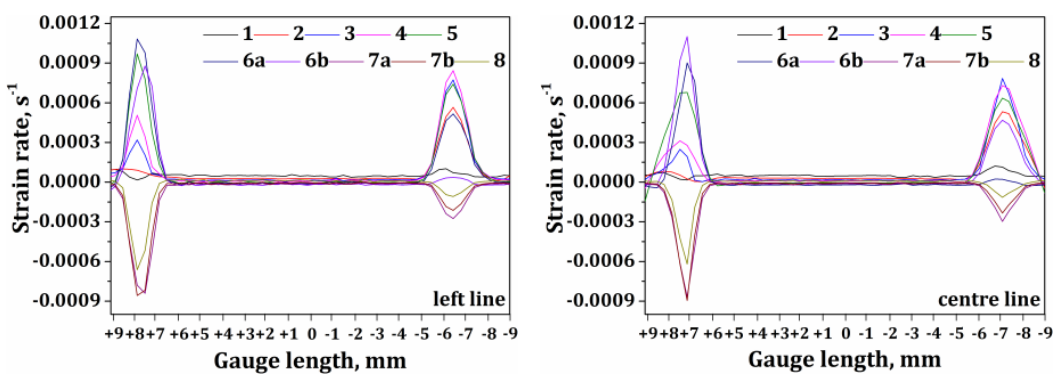

(a)

(b)

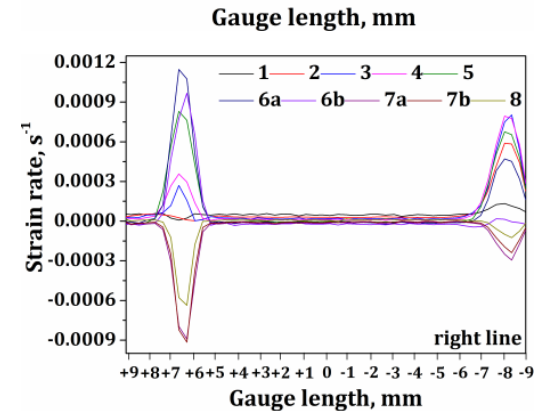

(c)

Figure. 6. Local strain (upper) and strain rate (lower) distributions along left (a), centre (b) and right (c) lines of the gauge length for cycle C4. The numbers and letters correspond to those in Fig. 5. 

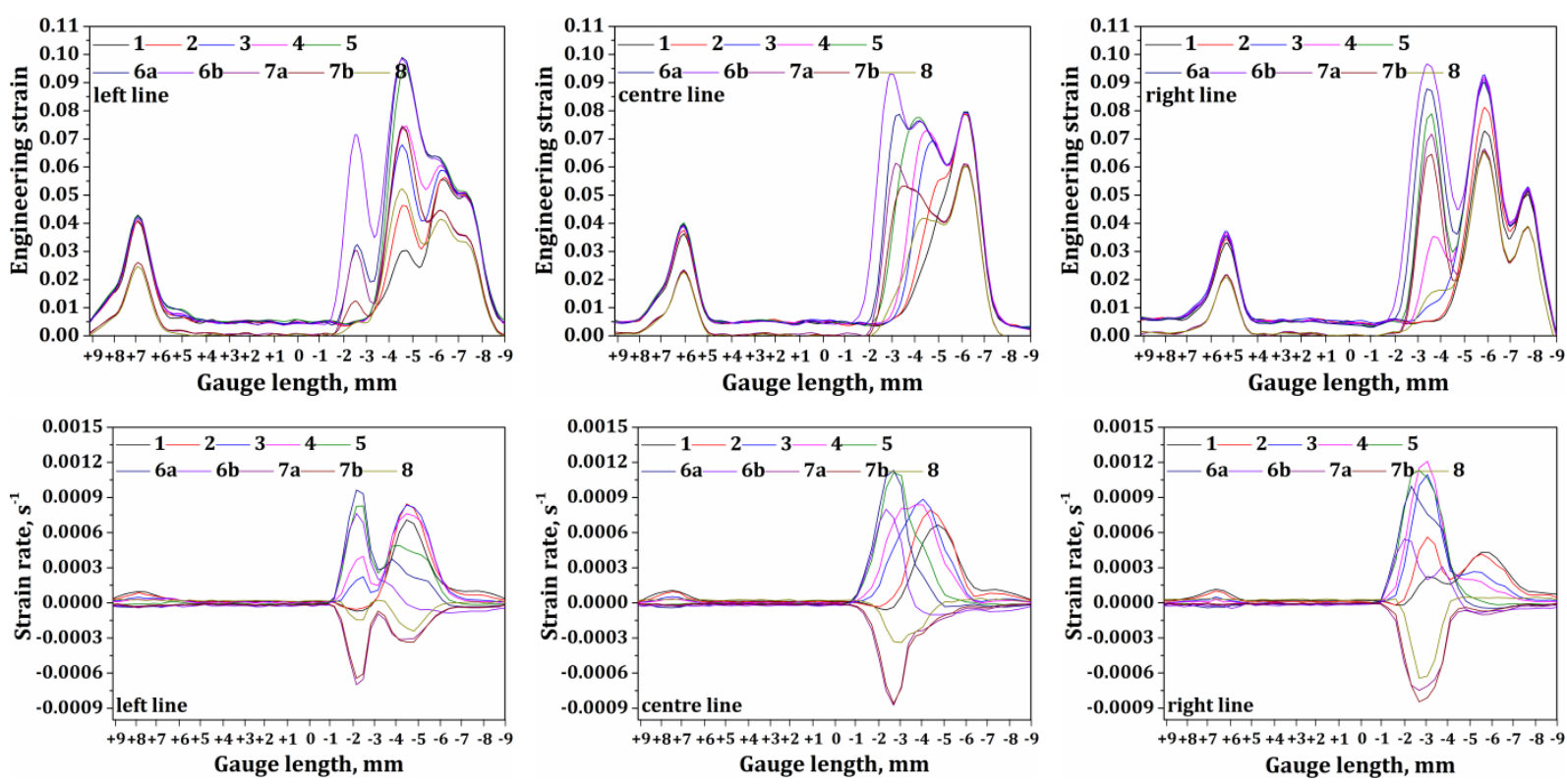

(a)

(b)

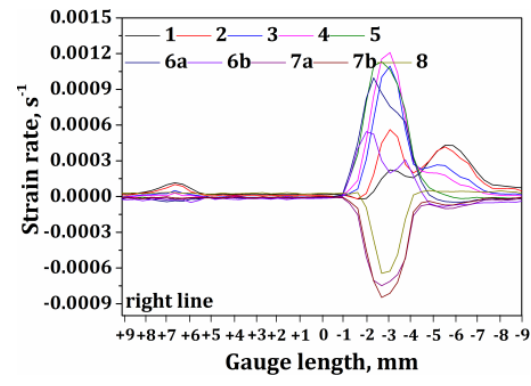

(c)

Figure. 7. Local strain (upper) and strain rate (lower) distributions along left (a), centre (b) and right (c) lines of the gauge length for cycle C7. The numbers and letters correspond to those in Fig. 5.

\section{Discussion}

\subsection{Factors affecting phase transformation during monotonic uniaxial tension}

For monotonic uniaxial tension, factors affecting phase transformation are discussed and the macroscopic stress versus strain response is compared with the literature. In polycrystalline NiTi alloys, phase transformation readily initiates in grains that are favourably oriented with respect to the macroscopic loading direction [1-4]. In the annealed material in the present work, the predominantly $\langle 111\rangle_{\mathrm{B} 2}$ grains of the B2 phase (inset in Fig. 8a) are suitably oriented for transformation to the B19' phase during tension $[4,12,30]$.

Brinson et al. [23] reported that as much as $\sim 30 \%$ by volume of unfavourably oriented B2 grains may remain potentially untransformed after the mobile front of a transformation band sweeps over a portion of the gauge length. It follows that the higher stresses needed to transform the remnant B2 grains within the band to $\mathrm{B} 19^{\prime}$ would result in large fluctuations in stress within the macroscopic stress plateau region. However, as shown in Fig. 1a, the macroscopic stress only fluctuates by a maximum of \pm 10 MPa within the macroscopic stress plateau region.

Furthermore, the propagation of transformation bands is intrinsically linked with latent heat release at their mobile fronts. When the sample thickness-to-length ratio or the loading speed is increased, the mobile front is unable to release latent heat sufficiently. This results in changes to the deformation accommodation behaviour such that single inclined band propagation gives way to multiple but similarly inclined bands, which then gives way to uniform strain along the gauge length $[1-4,13]$. In the present mechanical tests, a small 1:18 sample thickness-to-length ratio is coupled with 
a slow crosshead speed of $0.48 \mathrm{~mm} \cdot \mathrm{min}^{-1}$, which corresponds to an initial strain rate of $1 \times 10^{-4}$ $\mathrm{s}^{-1}$.

Consequently, favourably oriented $\langle 111\rangle_{\mathrm{B} 2}$ grains, a small sample thickness-to-length ratio and a slow crosshead speed result in a stress fluctuation of $\pm 10 \mathrm{MPa}$ within the macroscopic stress plateau region and a nominal transformation strain of $0.088^{1}$ [4].

The small stress fluctuation indicates ease in the propagation of a single transformation band along the entire gauge length without necessitating the development of multiple transformation bands. On the other hand, the large value of nominal transformation strain suggests the extensive phase transformation of $\mathrm{B} 2$ to $\mathrm{B} 19^{\prime}$ within the transformation band.

\subsection{Deviation from elastic linearity during mechanical testing}

As shown in Fig. 1a(inset), the deviation from linearity towards the end of the elastic region is followed by a stress reduction and an extended macroscopic stress plateau region [1, 31, 32]. Brinson et al. [23] provided in-situ optical microscopy evidence showing that at $\sim 0.007$ macroscopic strain, which is prior to the onset of the macroscopic stress plateau region, B19' variants form within isolated B2 grains throughout the gauge length. Consequently, the deviation from elastic linearity indicates the initiation of phase transformation within B2 grains that are preferentially oriented with respect to the macroscopic loading direction.

Fig. 1a and cycle C1 in Fig. 1b indicate that the B2 phase becomes unstable towards the end of the elastic region [2]. The stress enhancement provided by the geometry of the dog-bone samples leads to local stress accumulations near the ends of the gauge length, which in turn provides the activation energy for stress-induced transformation to $\mathrm{B}^{\prime} 9^{\prime}$. Once B19' forms, the local strain accumulations propagate across the sample width, resulting in a transformation band and stress relaxation/reduction in the sample bulk. The subsequent forward propagation of the band upwards and downwards along the gauge length and the ongoing phase transformation of remnant B2 grains within the bands results in stress fluctuations in the macroscopic stress plateau region. This phenomenon is clearly seen in points (c) to (3) in Fig. 2, points (4) to (6a) in cycle C2 in Fig. 5a.

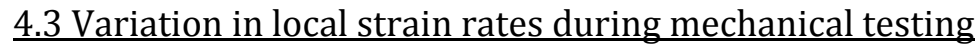

A study of the changes in local strain rates within Lüders bands of a Mg alloy subjected to tension [33] found that the maximum local strain rate was located at the mobile front of the propagating band. Schaefer and Wagner [3] performed monotonic uniaxial tension on a NiTi alloy and studied the local strain rate at one select macroscopic strain within the macroscopic stress plateau region. They showed that the maximum local strain rate is one order of magnitude higher than the macroscopic strain rate.

\footnotetext{
${ }^{1}$ This value is calculated by subtracting the macroscopic strain at maximum stress (associated with the onset of transformation) from the macroscopic strain at the end of the macroscopic stress plateau region (i.e. - the macroscopic strain at points 2 and 4 in Fig. 1a).
} 
Kim and Daly [9] studied the individual velocities of multiple propagating bands during tension of NiTi alloy. Given constant mechanical testing parameters, they found that regardless of the number of propagating bands that form during a test, the sum of the maximum velocities of individual bands resulted in approximately the same value as that of a single band.

This study expands the findings in Ref. [3] by extracting the maximum local strain rates for all time steps in both types of tests. Their evolution with macroscopic strain is studied. In this regard, the present mechanical tests show a single transformation band during monotonic uniaxial tension (Fig. 2), two transformation bands in cycle C4 (Fig. 5c) and up to six transformation bands in cycle C7 (Fig. 5e). Moreover, within the macroscopic stress plateau region, the maximum local strain rates of individual bands during cyclic loading in tension are lower than the maximum local strain rate during monotonic uniaxial tension. However, and analogous to Ref. [9], we find that the sum of the maximum local strain rates of individual bands during cyclic loading $\left(1.3-1.9 \times 10^{-3} \mathrm{~s}^{-1}\right)$ is approximately the same value as the maximum local strain rates of the single transformation band during monotonic uniaxial tension $\left(1.4-2.3 \times 10^{-3} \mathrm{~s}^{-1}\right)$. Consequently, we propose that the maximum local strain rate within the macroscopic stress plateau region may also be applied as a descriptive indicator to understand the influence of loading rate on superelastic behaviour and phase transformation in strain rate-dependent studies. On the other hand, the sum of the maximum local strain rates of individual bands during cyclic unloading is less, compared with its counterpart during loading (Figs. 6 and 7). However, it should be noted that in this test condition, the unloading stress versus strain curve is a combined response due to the elastic unloading of the B2 and B19' phases, and reverse transformation of B19' back to B2. Consequently, the maximum strain rates of individual transformation bands upon unloading are not comparable with that upon loading; the latter are extracted from regions where only transformation of $\mathrm{B} 2$ to $\mathrm{B} 19^{\prime}$ is occurring (within the mobile band fronts).

\subsection{Criss-cross transformation band formation during cyclic loading-unloading in tension}

The observation of: (i) a widening of a single inclined transformation band front and (ii) the generation of multiple criss-crossing transformation bands, provides insights into the mechanism of phase transformation under the applied test conditions. Kinking and straightening of the sample edge is rationalised with the build-up of an asymmetric in-plane moment due to local shear strains near the band front; the latter was suggested in Ref. [19].

As seen in Fig. 4a, the mobile, upper front of the transformation band is inclined at angle $(\eta)$ of $\sim 60^{\circ}$ to the sample axial length such that at 0.0264 macroscopic strain, a local $\sim 2^{\circ}$ kink is observed. On the other hand, the macroscopic stress plateau region of cycle C5 results in the development and propagation of a new transformation band inclined at $\sim 60^{\circ}$ criss-crossing the pre-existing bottom transformation band inclined at $\sim 120^{\circ}$ (Fig. 5d). This results in stress relaxation in the sample bulk, which is recorded as a reduction in the macroscopic stress prior to the onset of a new macroscopic stress plateau (Fig. 1b). 
In the case of monotonic uniaxial tension, the local shear strain at the mobile front of the transformation band leads to kinking of the gauge length as the magnitude of the moment increases. The disappearance of the kink is associated with a moment relieving mechanism and is accompanied by: (i) a widening of the mobile, upper front of the transformation band and, (ii) a straightening of the sample edge.

In the case of the cyclic loading-unloading, when the moment with inclination angle $\eta$ reaches a local maximum value, the sample is unable to accommodate it any further. Consequently, the moment relieving mechanism involves the appearance of a new band whose characteristic moment is of an opposite sign $(-\eta)$ [19]. The criss-crossing pattern forms because of the new band forming at the mobile front of a pre-existing band resulting in phase transformation of highly locally strained B2 to B19'. This is accompanied by: (i) stress fluctuations in the macroscopic stress versus strain curve and, (ii) a straightening of the sample edge.

\subsection{Stress increases and residual strain upon unloading}

In Fig. $1 \mathrm{~b}$ a stress increment is seen for cycles $\mathrm{C} 2$ to $\mathrm{C} 4$ during unloading and is indicative of reverse phase transformation of B19' to B2. During unloading, the anisotropic changes to the local stress fields surrounding low-symmetry monoclinic B19' lead to the early reverse transformation of particular variants to B2 while hindering the later reverse transformation of other variants. In turn, the latter requires an increase in stress in order to overcome the residual strain and reverse transform back to B2 [2].

The literature on fatigue tests has suggested that during unloading, the formation of stable remnant B19' variants, along with de-twinning, grain reorientation effects and the retention of lattice defects within B2 and remnant B19' grains hinder the latter phase's reverse transformation back to B2 $[14,15,22-28]$. The combined effect of the above mechanisms serves to increase the magnitude of residual strains upon unloading after each cycle along with a progressive degradation in superelasticity.

The EBSD work in this study evidences the kernel average misorientation of the B2 and remnant B19' grains after deformation and links the microstructure changes to the mechanical behaviour and pattern evolution of the transformation band(s) during testing. Consequently, the KAM maps of the present NiTi alloy before mechanical testing (Fig. 8b), upon unloading after monotonic uniaxial tension up to point 5 in Fig. 1a (Fig. 8d) and upon unloading after 20 cycles of loading-unloading in tension (Fig. 8f) are compared. The KAM criterion was selected as a mean to qualitatively depict the variation in plastic strain and the correlated geometrically necessary dislocation activity [34]. It is evident that the microstructure before mechanical testing comprises a single B2 phase with low to no intragranular local misorientation $\left(\leq 0.5^{\circ}\right)$ within the grains. After mechanical testing, the retention of remnant B19' grains is apparent. Upon unloading after monotonic uniaxial tension, the phase area fractions with intragranular local misorientation larger than $0.5^{\circ}$ are similar in both phases, with 
0.265 for B2 and 0.273 for $\mathrm{B}^{1} 9^{\prime}$. On the other hand, a slightly higher 0.384 area fraction was returned for the B19' phase compared to the 0.275 area fraction for the B2 phase after 20 loading-unloading cycles in tension (Fig. 8g).

Localised high KAM values $\left(\geq 1^{\circ}\right)$ are noted for: (i) the interiors of original B2 grains, (ii) areas that reverse transformed back to B2 upon unloading, (iii) the remnant B19' grains and, (iv) the interface between B2 and B19' phases. It can be surmised that the high KAM values in the original B2 grains is indicative of dislocation and twinning activity which in turn, leads to the retention of particular B19' variants upon unloading [34].

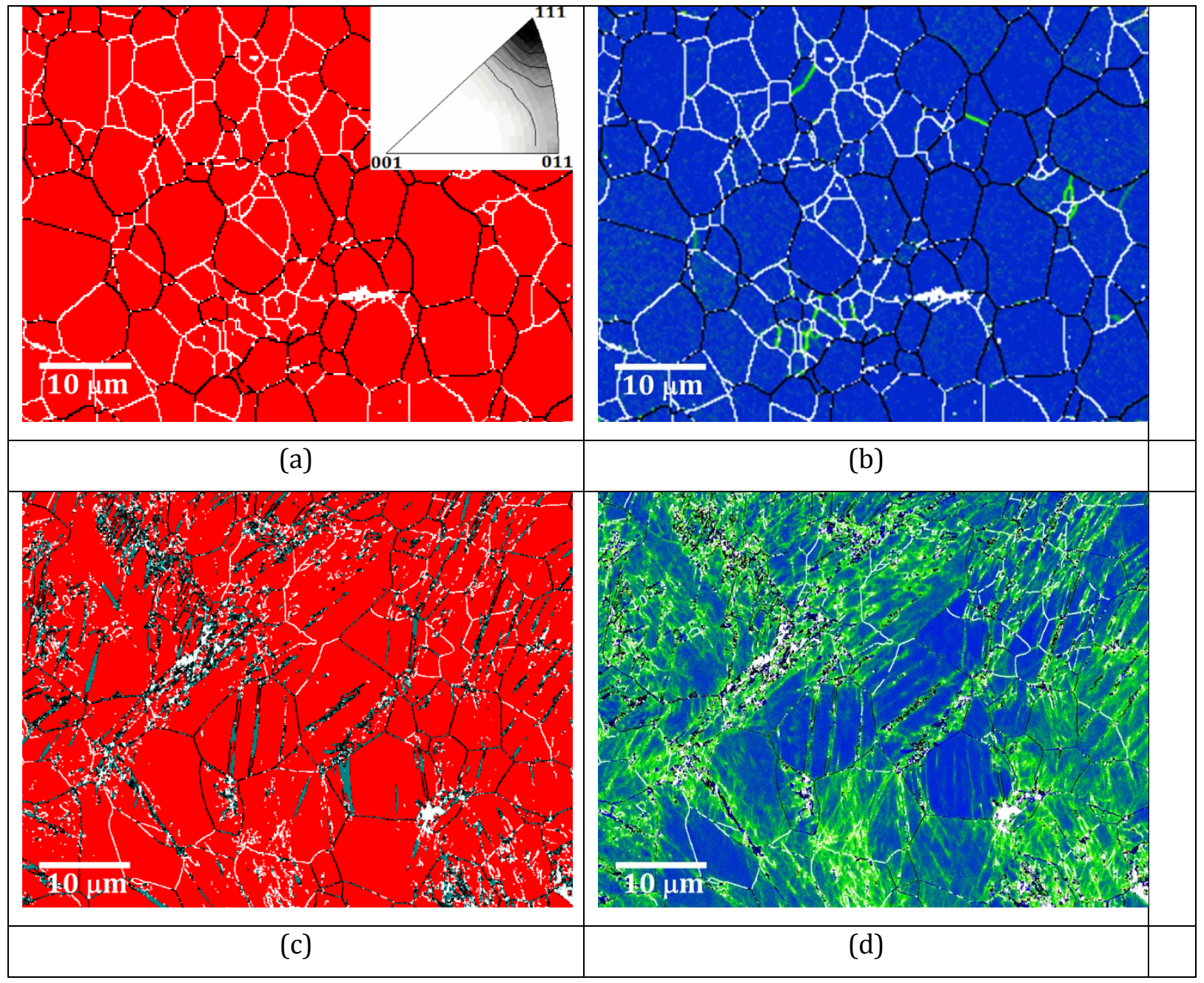




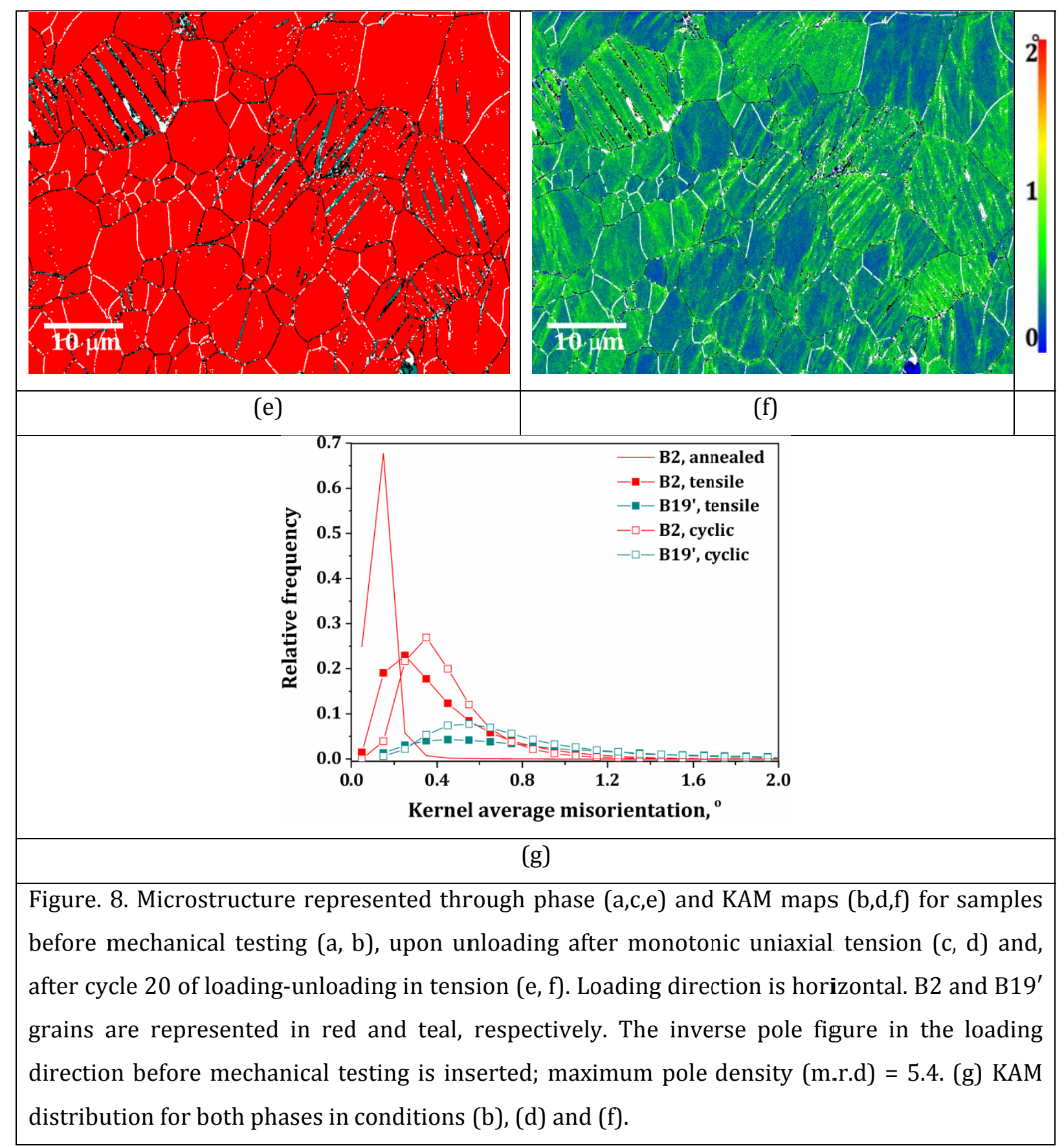

\section{Conclusions}

Using DIC, the local axial/shear strain fields and strain rates were studied during two types of mechanical tests involving monotonic uniaxial and cyclic loading-unloading in tension of a $56 \mathrm{Ni}-44 \mathrm{Ti}$ wt.\% shape memory alloy as follows:

1. The deviation from linearity in the macroscopic stress-strain curve suggests the initiation of B2 to B19' transformation. During monotonic uniaxial tension, the favourably oriented $\langle 111\rangle_{\mathrm{B} 2}$ grains, a small sample thickness-to-length ratio and a slow crosshead speed contribute to a relatively small stress fluctuation of $\pm 10 \mathrm{MPa}$ within the macroscopic stress plateau region and a relatively large nominal transformation strain of 0.088 . 
2. When the sample deforms uniformly, the maximum local strain rate $\dot{\varepsilon}_{M}$ is equivalent to the macroscopic strain rate. The formation of transformation band is reflected as a sharp increase of $\dot{\varepsilon}_{\mathrm{M}}$ that is 40 times higher than the macroscopic strain rate. During forward transformation in both tests, the sum of $\dot{\varepsilon}_{\mathrm{M}}$ of individual bands is approximately equivalent and is one order of magnitude higher than the macroscopic strain rate.

3. Broadened transformation band front and crossing patterns occur during monotonic uniaxial and cyclic loading-unloading in tension, respectively. This relieves the in-plane moment due to local shear strains and straightens the sample edge.

4. The high KAM values in the original B2 grains indicate dislocation and twinning activity, which in turn leads to the retention of particular B19' variants upon unloading. For cyclic loadingunloading test, this causes greater strain accommodation within pre-existing transformation bands and non-linearity for subsequent loading cycles.

\section{Acknowledgements}

This work was supported by the Australian Research Council - Discovery Project grant number DP130101883. This research also used equipment funded by the Australian Research Council Linkage, Infrastructure, Equipment and Facilities grant number LE0882613 located at the UOW Electron Microscopy Centre. The authors are grateful to the Nitinol Devices and Components Inc. and A/Prof. A. Stebner, Colorado School of Mines for providing the shape memory alloy. The authors thank Prof. Cheng Lu, Dr. Guillaume Michal and Mr. Jintao Li, UOW for their assistance with the DIC experiments and the Matlab script for sequential image analysis. 


\section{References}

[1] S. Daly, G. Ravichandran, K. Bhattacharya, Stress-induced martensitic phase transformation in thin sheets of Nitinol, Acta Materialia 55(10) (2007) 3593-3600.

[2] X. Huang, Y. Liu, Effect of annealing on the transformation behavior and superelasticity of NiTi shape memory alloy, Scripta Materialia 45(2) (2001) 153-160.

[3] A. Schaefer, M.F.-X. Wagner, Strain mapping at propagating interfaces in pseudoelastic NiTi, European Symposium on Martensitic Transformations (2009) 06031.

[4] P. Sittner, Y. Liu, V. Novak, On the origin of Lüders-like deformation of NiTi shape memory alloys, Journal of the Mechanics and Physics of Solids 53(8) (2005) 1719-1746.

[5] M.L. Young, M.F.X. Wagner, J. Frenzel, W.W. Schmahl, G. Eggeler, Phase volume fractions and strain measurements in an ultrafine-grained NiTi shape-memory alloy during tensile loading, Acta Materialia 58(7) (2010) 2344-2354.

[6] J. Mohd Jani, M. Leary, A. Subic, M.A. Gibson, A review of shape memory alloy research, applications and opportunities, Materials \& Design (1980-2015) 56(Supplement C) (2014) 1078-1113.

[7] M.A. Sutton, J.-J. Orteu, H. Schreier, Image Correlation for Shape, Motion and Deformation Measurements: Basic Concepts,Theory and Applications, Springer Publishing Company, Incorporated2009.

[8] J. Olofsson, I.L. Svensson, P. Lava, D. Debruyne, Characterisation and investigation of local variations in mechanical behaviour in cast aluminium using gradient solidification, Digital Image Correlation and finite element simulation, Materials \& Design (1980-2015) 56(Supplement C) (2014) 755-762.

[9] K. Kim, S. Daly, Martensite Strain Memory in the Shape Memory Alloy Nickel-Titanium Under Mechanical Cycling, Experimental Mechanics 51(4) (2011) 641-652.

[10] D. Saletti, S. Pattofatto, H. Zhao, Measurement of phase transformation properties under moderate impact tensile loading in a NiTi alloy, Mechanics of Materials 65(Supplement C) (2013) 1-11.

[11] Y. Xiao, P. Zeng, L. Lei, H. Du, Local Mechanical Response of Superelastic NiTi Shape-Memory Alloy Under Uniaxial Loading, Shape Memory and Superelasticity 1(4) (2015) 468-478.

[12] Y. Xiao, P. Zeng, L. Lei, Y. Zhang, In situ observation on temperature dependence of martensitic transformation and plastic deformation in superelastic NiTi shape memory alloy, Materials \& Design 134(Supplement C) (2017) 111-120.

[13] X. Zhang, P. Feng, Y. He, T. Yu, Q. Sun, Experimental study on rate dependence of macroscopic domain and stress hysteresis in NiTi shape memory alloy strips, International Journal of Mechanical Sciences 52(12) (2010) 1660-1670.

[14] L. Zheng, Y. He, Z. Moumni, Investigation on fatigue behaviors of NiTi polycrystalline strips under stress-controlled tension via in-situ macro-band observation, International Journal of Plasticity 90(Supplement C) (2017) 116-145. 
[15] X. Xie, Q. Kan, G. Kang, F. Lu, K. Chen, Observation on rate-dependent cyclic transformation domain of super-elastic NiTi shape memory alloy, Materials Science and Engineering: A 671(Supplement C) (2016) 32-47.

[16] D. Jiang, S. Kyriakides, C.M. Landis, Propagation of phase transformation fronts in pseudoelastic NiTi tubes under uniaxial tension, Extreme Mechanics Letters 15(Supplement C) (2017) 113-121.

[17] J.A. Shaw, S. Kyriakides, On the nucleation and propagation of phase transformation fronts in a NiTi alloy, Acta Materialia 45(2) (1997) 683-700.

[18] B. Reedlunn, C.B. Churchill, E.E. Nelson, J.A. Shaw, S.H. Daly, Tension, compression, and bending of superelastic shape memory alloy tubes, Journal of the Mechanics and Physics of Solids 63(Supplement C) (2014) 506-537.

[19] D. Jiang, S. Kyriakides, C.M. Landis, K. Kazinakis, Modeling of propagation of phase transformation fronts in NiTi under uniaxial tension, European Journal of Mechanics - A/Solids 64 (2017) 131-142.

[20] S.C. Mao, J.F. Luo, Z. Zhang, M.H. Wu, Y. Liu, X.D. Han, EBSD studies of the stress-induced B2-B19' martensitic transformation in NiTi tubes under uniaxial tension and compression, Acta Materialia 58(9) (2010) 3357-3366.

[21] E.A. Pieczyska, S.P. Gadaj, W.K. Nowacki, H. Tobushi, Phase-Transformation Fronts Evolution for Stress- and Strain-Controlled Tension Tests in TiNi Shape Memory Alloy, Experimental Mechanics 46(4) (2006) 531-542.

[22] O. Ammar, N. Haddar, L. Dieng, Experimental investigation of the pseudoelastic behaviour of NiTi wires under strain- and stress-controlled cyclic tensile loadings, Intermetallics 81(Supplement C) (2017) 52-61.

[23] L.C. Brinson, I. Schmidt, R. Lammering, Stress-induced transformation behavior of a polycrystalline NiTi shape memory alloy: micro and macromechanical investigations via in situ optical microscopy, Journal of the Mechanics and Physics of Solids 52(7) (2004) 1549-1571.

[24] R. Delville, B. Malard, J. Pilch, P. Sittner, D. Schryvers, Transmission electron microscopy investigation of dislocation slip during superelastic cycling of Ni-Ti wires, International Journal of Plasticity 27(2) (2011) 282-297.

[25] T. Ezaz, J. Wang, H. Sehitoglu, H.J. Maier, Plastic deformation of NiTi shape memory alloys, Acta Materialia 61(1) (2013) 67-78.

[26] Q. Kan, G. Kang, Constitutive model for uniaxial transformation ratchetting of super-elastic NiTi shape memory alloy at room temperature, International Journal of Plasticity 26(3) (2010) 441-465.

[27] S. Miyazaki, T. Imai, Y. Igo, K. Otsuka, Effect of cyclic deformation on the pseudoelasticity characteristics of Ti-Ni alloys, Metallurgical Transactions A 17(1) (1986) 115-120.

[28] L. Zheng, Y. He, Z. Moumni, Effects of Lüders-like bands on NiTi fatigue behaviors, International Journal of Solids and Structures 83(Supplement C) (2016) 28-44.

[29] J. Treadway, A. Abolmaali, F. Lu, P. Aswath, Tensile and fatigue behavior of superelastic shape memory rods, Materials \& Design 86(Supplement C) (2015) 105-113. 
[30] P. Šittner, P. Lukáš, V. Novák, M.R. Daymond, G.M. Swallowe, In situ neutron diffraction studies of martensitic transformations in NiTi polycrystals under tension and compression stress, Materials Science and Engineering: A 378(1) (2004) 97-104.

[31] Y. Liu, D. Favier, L. Orgéas, Influence of Elastic Energy on the Unloading Behaviour of NiTi Shape Memory Alloys, J. Phys. IV France 05(C8) (1995) C8-593-C8-598.

[32] G. Tan, Y. Liu, Comparative study of deformation-induced martensite stabilisation via martensite reorientation and stress-induced martensitic transformation in NiTi, Intermetallics 12(4) (2004) 373381.

[33] J. Min, L.G. Hector, J. Lin, J.T. Carter, A.K. Sachdev, Spatio-temporal characteristics of propagative plastic instabilities in a rare earth containing magnesium alloy, International Journal of Plasticity 57(Supplement C) (2014) 52-76.

[34] K.S. Suresh, S.K. Bhaumik, S. Suwas, Effect of thermal and thermo-mechanical cycling on the microstructure of Ni-rich NiTi shape memory alloys, Materials Letters 99(Supplement C) (2013) 150153. 